\title{
The inhibitory effects of probiotics on colon cancer cells: in vitro and in vivo studies
}

\author{
Fangjian Shang ${ }^{1 "}$, Xia Jiang ${ }^{1 "}$, Haobo Wang ${ }^{1}$, Shihao Chen ${ }^{1}$, Xin Wang ${ }^{2}$, Ying Liu ${ }^{1}$, Shang Guo ${ }^{1}$, Dongyun Li ${ }^{1}$, \\ Weifang Yu $^{3}$, Zengren Zhao ${ }^{1}$, Guiqi Wang ${ }^{1}$ \\ ${ }^{1}$ Department of General Surgery, Hebei Key Laboratory of Colorectal Cancer Precision Diagnosis and Treatment, The First Hospital of Hebei \\ Medical University, Shijiazhuang, China; ${ }^{2}$ Department of Pathology, The First Hospital of Hebei Medical University, Shijiazhuang, China; \\ ${ }^{3}$ Department of Endoscopy Center, The First Hospital of Hebei Medical University, Shijiazhuang, China \\ Contributions: (I) Conception and design: F Shang, G Wang, X Jiang; (II) Administrative support: X Jiang, W Yu, Z Zhao; (III) Provision of study \\ materials or funding: X Jiang, Z Zhao; (IV) Collection and assembly of data: F Shang, H Wang, S Chen, (V) Data analysis and interpretation: X \\ Wang, Y Liu, S Guo, D Li; (VI) Manuscript writing: All authors; (VII) Final approval of manuscript: All authors. \\ \#These authors contributed equally to this work. \\ Correspondence to: Zengren Zhao; Guiqi Wang. Department of General Surgery, Hebei Key Laboratory of Colorectal Cancer Precision Diagnosis \\ and Treatment, The First Hospital of Hebei Medical University, Donggang Road No. 89, Shijiazhuang, China. Email: zzr-doctor@163.com; \\ wangguiqi2011@126.com.
}

Background: Colorectal cancer (CRC) is the most common gastrointestinal malignancy. And probiotics may have the function of preventing colon cancer. The aim of this study was to investigate the inhibitory effects of a probiotic mixture on colorectal cancer and its potential mechanisms.

Methods: The effects of the probiotic mixture on proliferation and metastasis of mouse colon cancer CT26 cells were assessed by probiotics and cells co-culture assay, Cell Counting Kit-8 assay, colony formation assay, wound-healing assay, as well as migration and invasion assays. And CT26 cells were also transplanted into $\mathrm{BALB} / \mathrm{c}$ mice to construct transplanted tumor animal model. The mice were randomly divided into two groups, control group and probiotic mixture intragastric administration group, after injection 21 days the tumor size and infiltration of immune cells in the tumor or spleen tissues were analyzed by hematoxylin and eosin (HE) and immunohistochemistry (IHC) staining.

Results: The probiotic mixture significantly inhibited the proliferation, invasion, and migration ability of CT26 cells compare to the control cells $(\mathrm{P}<0.05)$. In the animal experiments, the tumor volume of mice that had been fed the probiotic mixture was significantly smaller than that of the control group $(\mathrm{P}<0.05)$. Compared with control mice, more apoptotic cells and infiltration of immune cells were showed in the tumor tissues of the mice treated with the probiotic mixture, and an increased number of CD8+ cells in the tumor and spleen tissues but no significant change in tissues.

Conclusions: These results suggested that the probiotic mixture could inhibit the growth of CT26 tumors and induce an immune response in vivo. The probiotic mixture also inhibited the invasion, migration, and proliferation ability of CT26 cells in vitro.

Keywords: Probiotic; CT26 cells; colorectal cancer (CRC)

Submitted Oct 28, 2020. Accepted for publication Dec 17, 2020.

doi: 10.21037/jgo-20-573

View this article at: http://dx.doi.org/10.21037/jgo-20-573 


\section{Introduction}

Colorectal cancer (CRC) is the most common gastrointestinal malignancy. It is the third most common cancer in the world and the second cause of cancer-related mortality (1). The occurrence of CRC is associated with many factors, including low physical activity, obesity and a high body mass index (BMI), high-fat and low-fiber diets, alcohol consumption, smoking, the use of antiinflammatory non-steroidal drugs, and family history (2). CRC is a type of solid tumor which tends to occur in older people, and surgery is the main form of treatment. In recent years, in addition to surgical treatment, the research and development of probiotics has provided novel therapies for the treatment of solid tumors.

Probiotics have been shown to have beneficial effects on human health, including antibacterial activity against intestinal pathogens, regulating blood cholesterol levels, reducing the incidence of colitis and inflammation, regulating the immune system, and preventing colon cancer (3). The International Scientific Association for Probiotics and Prebiotics (ISAPP) defined probiotics as "live microorganisms that, when administered in adequate amounts, confer a health benefit on the host" (4). Most probiotics are found in the natural intestinal flora, and the main component of probiotics is lactic acid bacteria (LAB). Lactobacillus and Bifidobacterium are the most common LAB. Many studies have shown that probiotics can treat a range of gastrointestinal diseases, such as acute diarrhea, antibiotic-associated diarrhea, functional digestive disorders, and inflammatory intestinal diseases, by affecting or even changing the intestinal flora (5). It can also participate in the dynamic balance and regulation of the intestinal epithelial system. Moreover, probiotics can affect the quality and quantitative composition of intestinal ecosystems (4).

Research has shown that the immunomodulatory and anti-tumor effects of probiotics are particularly remarkable. There has been increasing evidence to support the feasibility of immunotherapy as a promising therapeutic strategy for the treatment of solid tumors (6). One report showed that the appropriate regulation of intestinal microbiota through the intake of specific probiotics may help to prevent tumor formation (7). L. plantarum has been shown to inhibit the growth of CT26 colon carcinoma cells in subcutaneous tumor-bearing mice, most likely by changing the tumor microenvironment and promoting the migration of CD8 T cells and natural killer (NK) cells to the tumor tissue (8). Another study demonstrated that oral administration of
L. casei induced a potent $T$ helper 1 cell immune response and cytotoxic $\mathrm{T}$ cell infiltration in the tumor tissue of tumor-bearing mice, resulting in CT26 tumor growth inhibition (9).

This current study explored the inhibitory effects of a probiotic mixture on CT26 cells, both in vitro and in an animal model. Furthermore, the effects of the probiotic mixture on immune regulation were explored.

We present the following article in accordance with the ARRIVE reporting checklist (available at http://dx.doi. org/10.21037/jgo-20-573).

\section{Methods}

\section{Mice and ethics statement}

Female BALB/c mice were purchased from Hebei Medical University (Shijiazhuang, Hebei, China) at 6-8 weeks of age, weighing $20-25 \mathrm{~g}$, and acclimatized in Hebei Medical University. All animals received humane care. Animal experiments were reviewed and approved by the Animal Committee of the First Hospital of Hebei Medical University (License number 20200326) and guidelines for the care and use of animals were followed.

\section{Composition of the probiotic mixture}

A probiotic mixture was provided by Hebei Inatural Biotech CO., LTD for free, that containing 30 billion colony-forming units (CFU)/g was used in these experiments. The mixture was composed of $B$. longum, B. bifidum, L. acidophilus, L. plantarum, resistant dextrin, isomaltooligosaccharides, fructose oligosaccharides, and stachyose.

\section{Cell culture}

The mouse-derived CT26 colon cancer cell line was cultured in Roswell Park Memorial Institute (RPMI)-1640 medium (Gibco), supplemented with 10\% Fetal Bovine Serum (FBS; Gibco) and $1 \%$ penicillin-streptomycin (Invitrogen, Carlsbad, CA, USA) at $37{ }^{\circ} \mathrm{C}$ in a $5 \% \mathrm{CO}_{2}$ incubator, and maintained in exponential growth.

\section{Co-culture assay}

The CT26 cells were evenly seeded into 6-well plates at $1 \times 10^{5}$ cells per well. The cells were then cultured at 
$37{ }^{\circ} \mathrm{C}$ in a $5 \% \mathrm{CO}_{2}$ incubator for 14 hours to allow for cell adhesion to the wells. Cells in the probiotic mixture (PM) group were co-cultured with $2 \mathrm{~mL}$ of the probiotic mixture $\left(1 \times 10^{8} \mathrm{CFU} / 2 \mathrm{~mL}\right)$ at $37^{\circ} \mathrm{C}$ for 8 hours. The same amount of RPMI-1640 culture media supplemented with $10 \%$ FBS was added to the control (CTL) cells. Following co-culture for 8 hours, cells were harvested for further follow-up experiments.

\section{Cell Counting Kit-8 assay (CCK-8)}

Cell viability was assessed using the CCK-8 assay (Sigma Aldrich, Missouri, USA). CT26 cells in the PM group and the CTL group were seeded into 96-well plates at a density of 1,000 cells/well. At different time intervals of 24, 48, 72, and 96 hours, $10 \mu \mathrm{L}$ of CCK-8 solution (Dojindo, Kumamoto, Japan) was added to each well, and the cells were incubated for 1.5 hours at $37^{\circ} \mathrm{C}$. The absorbance was then measured using a Promega GloMax Luminescence detector at a wavelength of $450 \mathrm{~nm}$. The absorbance of the CT26 cells in the PM group and the CTL group was compared and used to create a proliferation curve.

\section{Colony formation assay}

For the colony formation assays, CT26 cells from the PM group and the CTL group were inoculated into $10 \mathrm{~mm}$ petri dishes (1,000 cells per dish) and cultured for 2 weeks. Subsequently, colonies were fixed with $4 \%$ phosphatebuffered formalin ( $\mathrm{pH}$ 7.4) and stained with Giemsa' staining solution (solarbio, Beijing, China) for 20 minutes. Colonies were assessed and quantified.

\section{Wound-healing assay}

Following probiotic treatment or control treatment, the CT26 cells were harvested and seeded into 6-well plates at a density of $1 \times 10^{6}$ cells/well. After 24 hours, a single scratch was made in the layer of cells using the tip of a $200 \mu \mathrm{L}$ fluid transfer gun. Cells were then rinsed with phosphate buffer saline (PBS). Photographs of cell migration across the scratched line were recorded at 0 and 48 hours using light microscopy.

\section{Migration and invasion assay}

Cell migration and invasion assays were performed using membranes with $8 \mu \mathrm{m}$ pores (BD Biosciences, Franklin
Lakes, NJ, USA). Prior to the assays, the chambers were taken out of the $-20{ }^{\circ} \mathrm{C}$ storage environment and placed at $37^{\circ} \mathrm{C}$ for 1 hour to allow the matrigel (354480; BD) to melt. Cells were resuspended in serum-free RPMI-1640 medium to give a suspension of $1 \times 10^{5}$ cells $/ 100 \mu \mathrm{L}$. In a 24-well plate, $650 \mu \mathrm{L}$ of $10 \%$ FBS RPMI-1640 medium was added to the bottom of the wells. The chambers were then gently transferred to the 24-well plate, and $100 \mu \mathrm{L}$ of cell suspension was evenly distributed onto the basement membrane of the chambers and incubated at $37^{\circ} \mathrm{C}$. The chambers were taken out after 24 hours and the medial cells of each chamber were carefully wiped with a cotton swab and fixed with $4 \%$ formaldehyde solution. Samples were then stained with hematoxylin and eosin violet. Cells on the outer membrane of the chambers were observed under a microscope ( $\times 200$ magnification), and the number of cells in 10 random fields of view were counted and averaged. Except for melting of the matrix, all other steps of the migration assay were identical to those of the invasion assay.

\section{Transplanted tumor mouse model and administration of the probiotic mixture}

Eight female BALB/c mice (6-8 weeks old, 20-25 g) were randomly separated into two groups with four mice in each group. To generate the transplanted tumor model, $1 \times 10^{6}$ CT2 6 cells were injected into the right groin of each mouse.

One group of mice were then administered the probiotic mixture $\left(1 \times 10^{9} \mathrm{CFU} / 200 \mu \mathrm{L}\right)$ by intragastric gavage, and the other group were administered the same volume of PBS. The former group was designated as the CT26 plus probiotic mixture (PM) group, and the latter was designated as the CT26 control group (CTL). All mice were raised in the same environment with adequate food and water.

During the experiment, the survival status of the mice was observed every day. At day 21, the mice were sacrificed by cervical dislocation. Tumor volume was measured using a digital vernier caliper and calculated using the following formula: $\mathrm{V}=1 / 2 \mathrm{~L} \times \mathrm{W}^{2}$, where $\mathrm{L}=$ length, and $\mathrm{W}=$ width (10).

\section{Hematoxylin \& eosin staining and immunobistochemistry}

After the mice were sacrificed at the designated time, tissue samples, including the tumor and the spleen, were harvested. Tissue samples were fixed in $10 \%$ buffered formalin, embedded in paraffin, and sectioned. The sections 
A

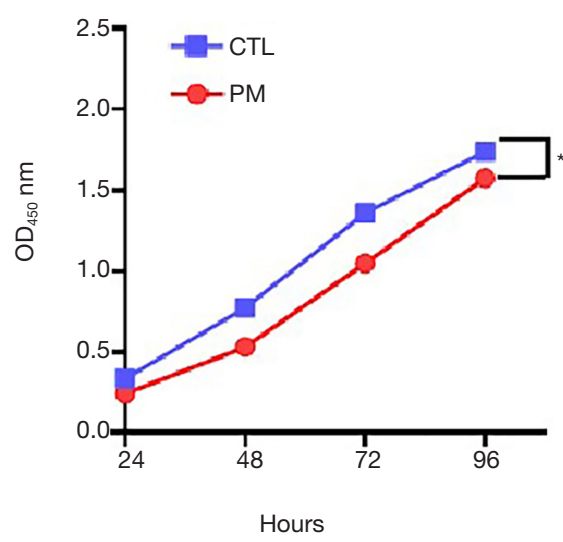

B

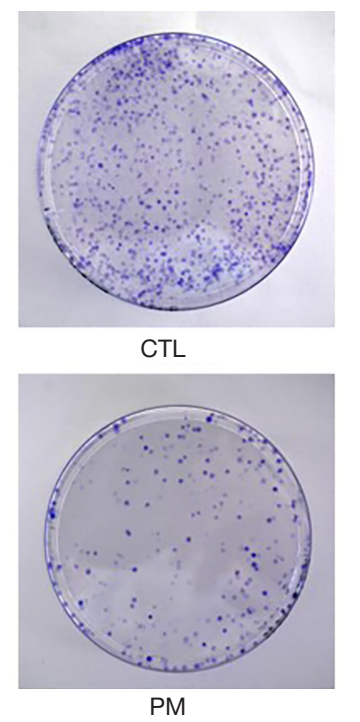

C

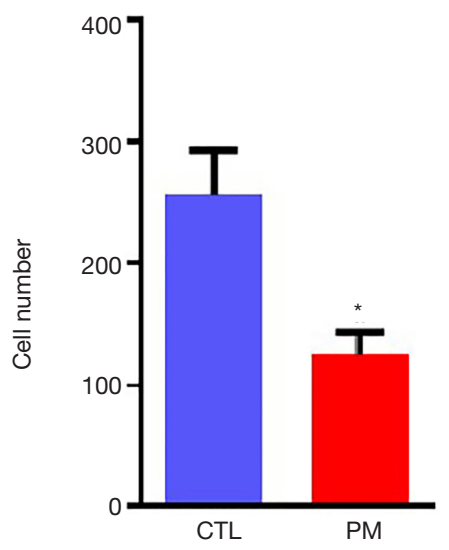

Figure 1 The inhibitory effects of the probiotic mixture on the proliferation of CT26 cells. (A) The OD values of the PM group and the CTL group were measured at 24, 48, 72, and 96 hours; (B) representative images of the number of CT26 cell colonies in the CTL group and the PM group; Figure $1 B$ showed a 10-cm diameter dish. (C) Diagram showing that the probiotic mixture inhibited colony formation of CT26 cells compared to the CTL group. *, $\mathrm{P}<0.05$. PM, probiotic mixture; CTL, control.

were stained with hematoxylin and eosin (HE) and observed under light microscopy.

Tissue sections were dewaxed and hydrated using a xylene and gradient alcohol series and rinsed with PBS for 5 minutes. The sections were then incubated in methanol or a $0.3 \%$ hydrogen peroxide solution for 30 minutes to quench the activity of the endogenous peroxidase, and washed in buffer for 5 minutes. Subsequently, the tissue was incubated at room temperature with diluted normal serum for 20 minutes (where possible, the source of the serum was consistent with the source of the secondary antibody). After blotting the excess serum, the sections were incubated with a primary antibody (CD8, 1:200, Abcam, Cambrige, MA, USA) for 30 minutes followed by a 5 -minute buffer wash. Sections were then incubated for 30 minutes with a diluted biotinylated secondary antibody (1:100, Abcam) solution and washed for 5 minutes in buffer. Next, sections were incubated for 30 minutes with VECTASTAIN ABC reagent (ZSGB-BIO, China) followed by a 5 -minute buffer wash. Lastly, the sections were incubated in peroxidase substrate solution until the desired stain intensity had developed. Slides were then rinsed under tap water.

\section{Statistical analysis}

SPSS 16.0 (SPSS Inc., Chicago, IL, USA) was used for data analysis. The measurement data were expressed as mean \pm standard deviation. $T$ tests were used for comparisons between two groups and analysis of variance (ANOVA) was used for comparisons between multiple groups. Statistical significance was set at $\mathrm{P}<0.05$.

\section{Results}

\section{The probiotic mixture inbibited CT26 cell proliferation}

Cell viability was assessed using the CCK- 8 assay kit. The absorbance of the cells was recorded at four different time points and the levels of cell proliferation were evaluated. The absorbance level of cells treated with the probiotic mixture was significantly lower than that of the control group, although the absorbance in both groups showed an increasing trend with time $(\mathrm{P}<0.05$; Figure $1 A)$. Furthermore, after 14 days, the colony formation assay revealed that the number of colonies in the PM group was significantly less than that in the CTL group $(\mathrm{P}<0.05$; Figure $1 B, C)$. These results demonstrated that the probiotic mixture had an effect on the long-term survival of CT26 cells.

\section{The probiotic mixture decreased the ability of migration and invasion of the CT26 cells}

As shown at Figure $2 A$ and $B$, compared with the CT26 cells 
A

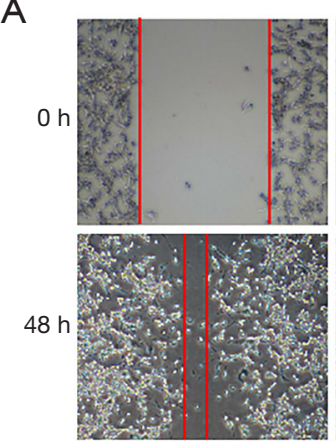

CTL

C

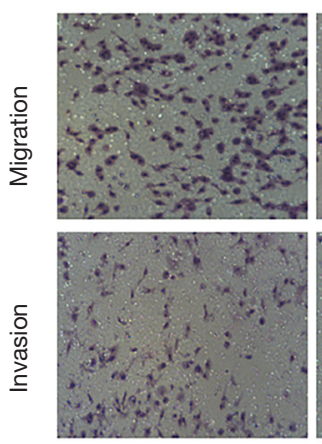

CTL

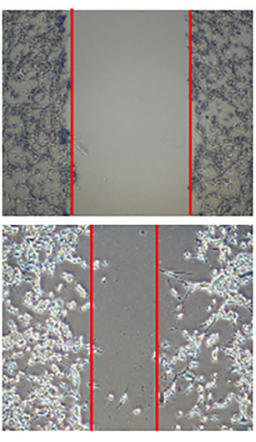

PM

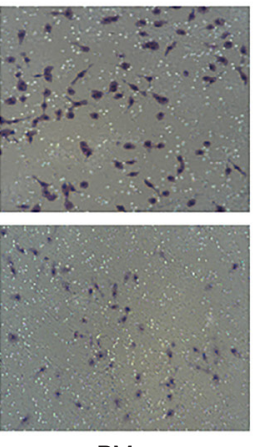

PM
B

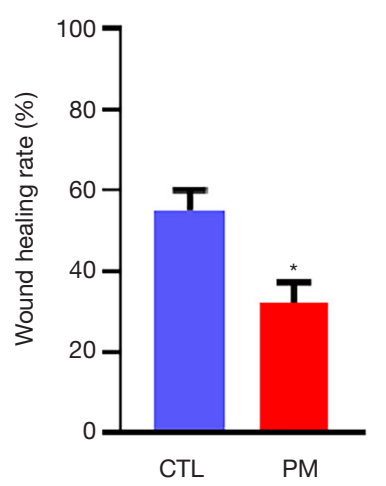

D

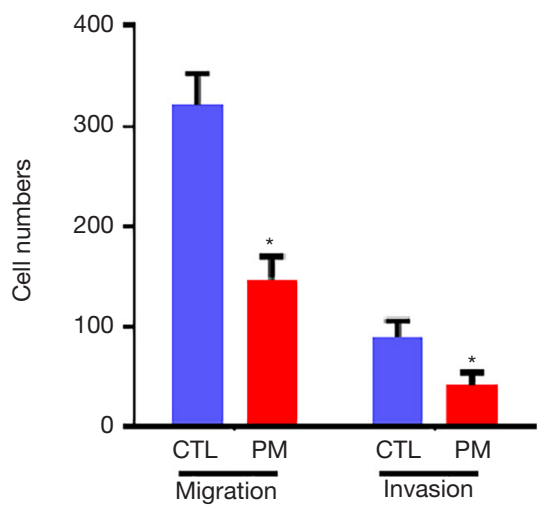

Figure 2 The probiotic mixture decreased the ability of migration and invasion of the CT26 cells. (A) The wound healing observed in CT26 cells in the CTL group and the PM group after 48 hours (400x); (B) a graph showing that the probiotic mixture suppressed the wound healing ability of CT26 cells; (C) cell migration and invasion of CT26 cells without and with the probiotic mixture (400×); (D) a graph showing that the probiotic mixture inhibited the migration and invasion capabilities of CT26 cells. * $\mathrm{P}<0.05$. PM, probiotic mixture; CTL, control.

in the control group, the wound healing efficiencies were reduced after treatment with the probiotic mixture $(\mathrm{P}<0.05)$. The results of the invasion and migration assays showed that the numbers of migrated and invasive CT26 cells in the PM group were significantly less compared with the CTL group (Figure $2 C, D ; \mathrm{P}<0.05$ ).

\section{The probiotic mixture inbibited the tumor growth in vivo}

The protective effect of the probiotic mixture against CT26-induced colon carcinoma was evaluated. On day $1,1 \times 10^{6}$ CT26 cells were injected into the right groin of each mouse. Mice in the PM group received a daily oral dose of the probiotic mixture $\left(1 \times 10^{9} \mathrm{CFU} / 200 \mu \mathrm{L}\right)$ and the CTL group was given $200 \mu \mathrm{L}$ PBS by gavage (Figure $3 A$ ). At day 21, mice from both groups were sacrificed and the tumors were isolated. As shown in Figure $3 B$ and C, the tumors in the PM group were significantly smaller than that in the CTL group. Measurement of the tumor volumes revealed that the tumor volume in the mice treated with the probiotic mixture were significantly suppressed compared to the control mice.

\section{The probiotic mixture inhibited the tumor invasion and increased tumor immunity}

The tumor tissues and spleens were sampled from both groups of CT26 tumor-bearing mice to determine whether the probiotic mixture treatment induced any histopathological changes. HE staining revealed that compared to the control, a higher number of apoptotic cells was observed in the tumors of mice treated with the probiotic mixture (Figure $4 A$ ). Furthermore, immunohistochemical analyses of CD8 expression, the 
A

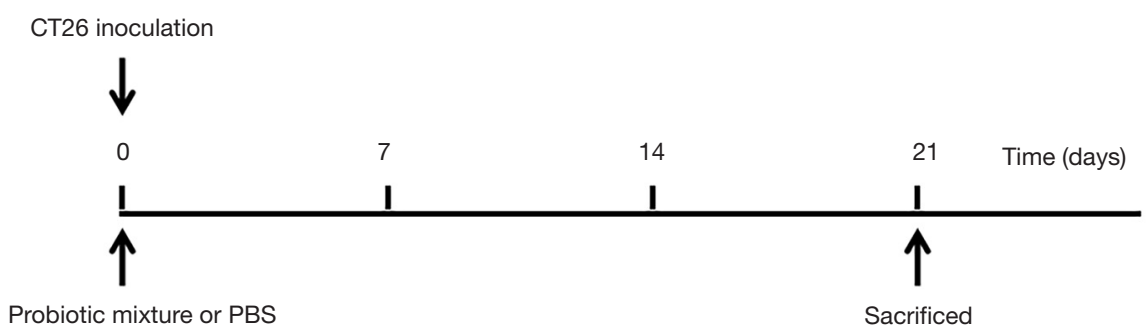

B

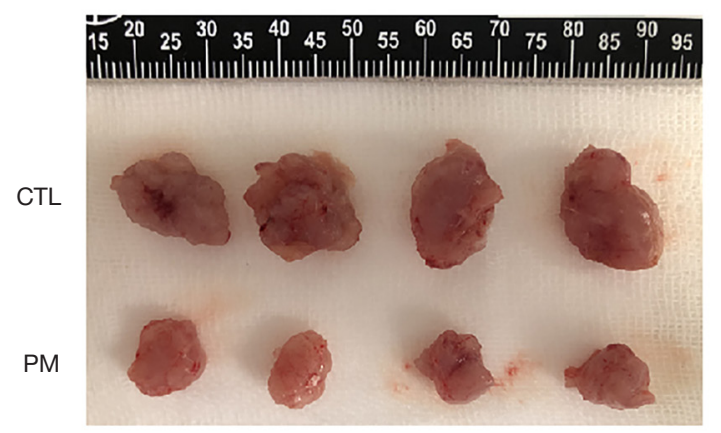

C

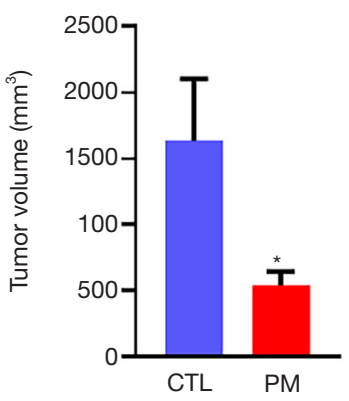

Figure 3 Oral administration of the probiotic mixture suppressed CT26 tumor growth in BALB/c mice in vivo. (A) $1 \times 10^{6}$ CT26 cells were injected into the right groin of BALB/c mice prior to administration of the probiotic mixture or PBS. All mice were sacrificed at day 21 by cervical dislocation; (B) image of tumors harvested from both groups of mice at day 21 ; (C) tumor volume in each group of mice at day 21. Graph shows tumor volume inhibition in mice fed with the probiotic mixture compared to control mice, ${ }^{*}, \mathrm{P}<0.05$.

expression of CD8 in tumor and spleen were stronger than that in control group (Figure 4B).

\section{Discussion}

CRC is a life-threatening disease and its rate of fatality remains high. The incidence of CRC has been associated with environmental and genetic factors, and has been linked with inflammatory bowel disease (IBD) and intestinal flora (11). Studies have shown that probiotics can regulate the microbiota of the intestinal tract and inhibit the progression of cancers (12). This suggests that there is a special connection between probiotics and CRC. In fact, numerous studies have demonstrated the anti-tumor effects of probiotics such as L. acidophilus, L. casei, L. plantarum, and B. longum $(13,14)$. However, before a specific probiotic strain can be considered part of a cancer treatment regimen, its function needs to be assessed in detail (15). In this study, a probiotic mixture, including $B$. longum, B. bifidum, $L$. acidophilus, and $L$ plantarum, was selected. The effects of this probiotic mixture on CT26 colon carcinoma cells were explored both in vivo and in vitro.

Tumor cells have a strong ability to invade, migrate and proliferate. Previous studies have shown that after incubation with probiotics, the proliferation of CRC cells was decreased (16). Probiotic derivatives and products were also shown to have the ability to inhibit the proliferation of CRC cells (17). In our experiment, the results demonstrated that the probiotic mixture significantly reduced the CT26 cell viabilities of proliferation and colony formation. after treatment with the probiotic mixture, CT26 cells wound healing degree, migration and invasion were also inhibited in this study. These results strongly suggested that this probiotic mixture had an inhibitory effect on CT26 cell proliferation and metastasis. This agreed with previous studies showing that probiotics could suppress the growth of CRCs through the inhibition of invasion, migration and the scratch healing processes $(18,19)$. Taken together, these results further support the notion that probiotic mixtures inhibit the ability of CRC cells to migrate and invade.

Furthermore, to examine the effects of the probiotic mixture on CT26-induced colon carcinoma, a CT26 transplanted tumor mouse model was established. In these in vivo experiments, oral administration of the probiotic mixture resulted in significantly smaller tumors compared to control mice, suggesting that the probiotic mixture 
A

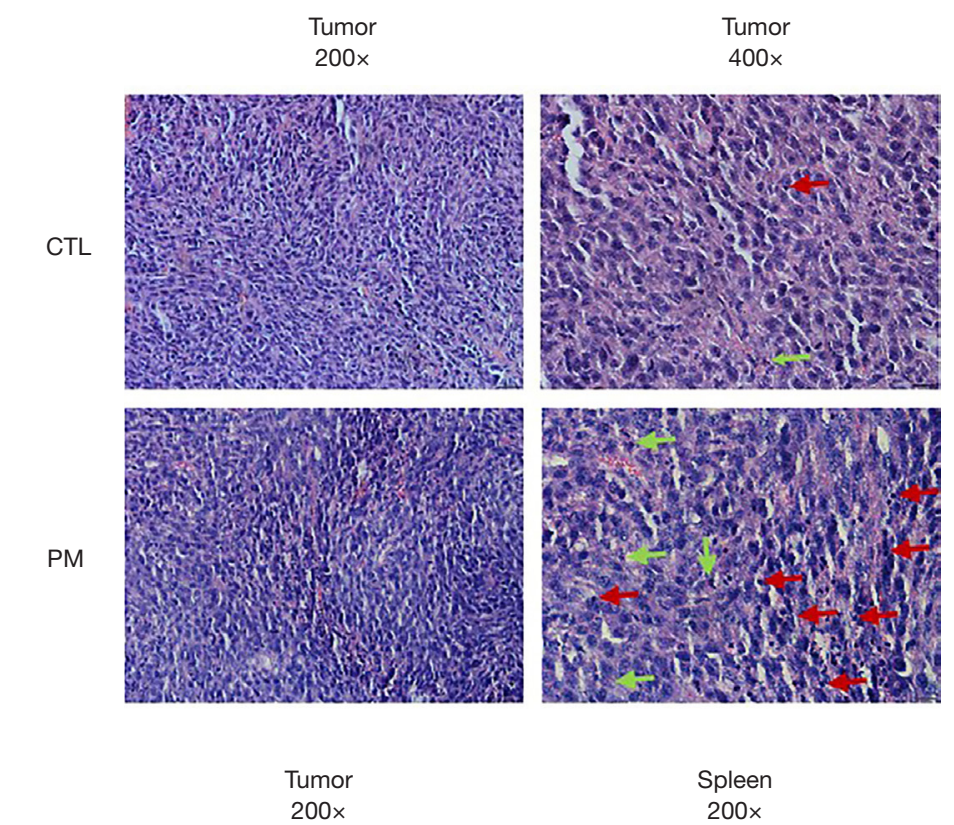

B

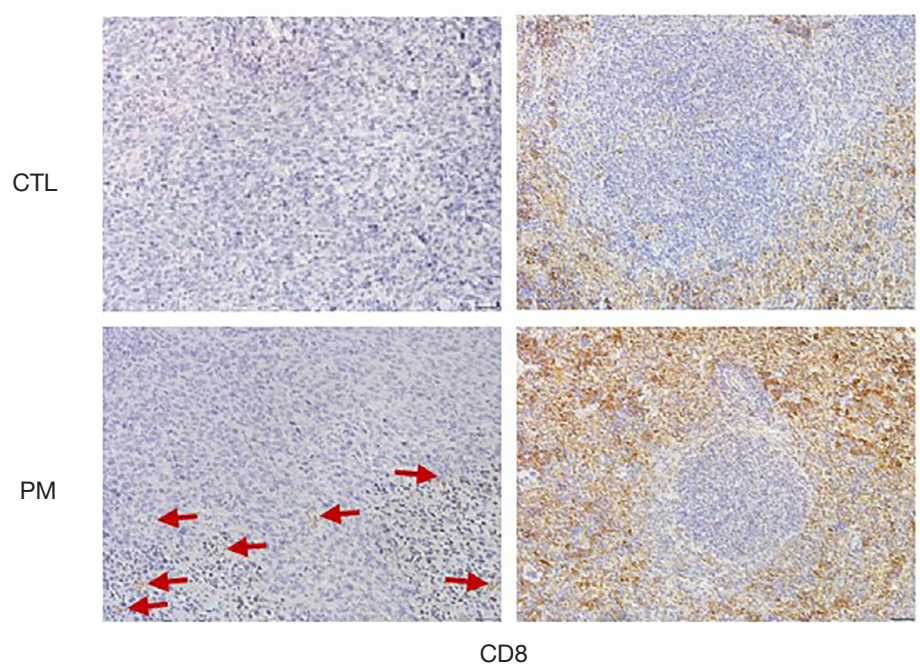

Figure 4 Representative HE and IHC in BALB/c mice tumor-bearing experiment. (A) Histopathological assessment with hematoxylin and eosin staining for tumors from both groups. Representative images of the necrosis of the tumors in each group of mice (200x or 400x magnification); the red arrows indicate tumour-infiltrating lymphocytes (TILs); the green arrows indicate apoptotic cell. (B) Representative images showing CD8 positive staining in the spleen tissues of mice treated with PBS control or the probiotic mixture (200× magnification).

significantly reduced tumor growth. Previous studies have demonstrated that many probiotics can enhanced the antitumor immune response and inhibit CT26 cell growth in BALB/c mice $(9,20)$.

To further verify whether the tumor-inhibitory effects of the probiotic mixture were related to the immune response, immunohistochemical analyses of the spleen were conducted. The numbers of CD8 positive cells in the tumor and spleen of probiotic-treated mice were significantly higher than that observed in the control group, indicating that the administration of the probiotic mixture resulted in the activation of more CD8 cells. These data demonstrated that the probiotic mixture induced $\mathrm{T}$ cell-mediated immune responses. Previous studies have shown that probiotics can regulate $\mathrm{T}$ lymphocytes to inhibit tumor growth (21). So, we consider that the anti-tumor effect of this probiotic 
mixture may be achieved by inducing $\mathrm{CD}^{+} \mathrm{T}$ cell immune response.

Herein, we demonstrated that the probiotic mixture used in this investigation activated more CD8 cells, and retarded and inhibited the growth of CT26 tumors. Moreover, the probiotic mixture also inhibited the invasion, migration, and proliferation capabilities of CT26 cells. Our results suggested that a variety of probiotics can work together to raise an immune response and inhibit tumor growth, and thus, probiotics may be an effective novel anticancer agent for future therapeutic regimens.

\section{Acknowledgments}

Funding: This work was supported by the Natural Science Foundation of Hebei (grant No. H2020206374), International Science and Technology Cooperation Program of Hebei (grant Nos. 2019YX006A and YZ201802), Foundation for Distinguished Young Talents in Higher Education of Hebei (grant No. BJ2018042 and LNB201911) and the Graduate Innovation Funding Project Foundation, Hebei, P.R. China (Grant No. CXZZBS2020111).

\section{Footnote}

Reporting Checklist: The authors have completed the ARRIVE reporting checklist. Available at http://dx.doi. org/10.21037/jgo-20-573

Data Sharing Statement: Available at http://dx.doi. org/10.21037/jgo-20-573

Conflicts of Interest: All authors have completed the ICMJE uniform disclosure form (available at http://dx.doi. org/10.21037/jgo-20-573). The authors have no conflicts of interest to declare.

Ethical Statement: The authors are accountable for all aspects of the work in ensuring that questions related to the accuracy or integrity of any part of the work are appropriately investigated and resolved. Animal experiments were reviewed and approved by the Animal Committee of the First Hospital of Hebei Medical University (License number 20200326) and guidelines for the care and use of animals were followed.

Open Access Statement: This is an Open Access article distributed in accordance with the Creative Commons Attribution-NonCommercial-NoDerivs 4.0 International License (CC BY-NC-ND 4.0), which permits the noncommercial replication and distribution of the article with the strict proviso that no changes or edits are made and the original work is properly cited (including links to both the formal publication through the relevant DOI and the license). See: https://creativecommons.org/licenses/by-nc-nd/4.0/.

\section{References}

1. Bray F, Ferlay J, Soerjomataram I, et al. Global cancer statistics 2018: GLOBOCAN estimates of incidence and mortality worldwide for 36 cancers in 185 countries. CA Cancer J Clin 2018;68:394-424.

2. Ulaganathan V, Kandiah M, Mohd Shariff Z . A casecontrol study of the association between metabolic syndrome and colorectal cancer: a comparison of International Diabetes Federation, National Cholesterol Education Program Adults Treatment Panel III, and World Health Organization definitions. J Gastrointest Oncol 2018;9:650-663.

3. Hill C, Guarner F, Reid G, et al. Expert consensus document. The International Scientific Association for Probiotics and Prebiotics consensus statement on the scope and appropriate use of the term probiotic. Nat Rev Gastroenterol Hepatol 2014;11:506-14.

4. Molska M, Regua J. Potential Mechanisms of Probiotics Action in the Prevention and Treatment of Colorectal Cancer. Nutrients 2019;11:2453.

5. Liévin-Le Moal V, Servin AL. Anti-infective activities of lactobacillus strains in the human intestinal microbiota: from probiotics to gastrointestinal antiinfectious biotherapeutic agents. Clin Microbiol Rev 2014;27:167-99.

6. Yang Y. Cancer immunotherapy: harnessing the immune system to battle cancer. J Clin Invest 2015;125:3335-7.

7. Genaro SC, Reis L, Reis SK, et al. Probiotic supplementation attenuates the aggressiveness of chemically induced colorectal tumor in rats. Life Sci 2019;237:116895.

8. Hu J, Wang C, Ye L, et al. Anti-tumour immune effect of oral administration of Lactobacillus plantarum to CT26 tumour-bearing mice. J Biosci 2015;40:269-79.

9. Aindelis G, Tiptiri-Kourpeti A, Lampri E, et al. Immune Responses Raised in an Experimental Colon Carcinoma Model Following Oral Administration of Lactobacillus casei. Cancers (Basel) 2020;12:368. 
10. Owyang SY, Zhang M, Owyang CC, et al. The effect of CT26 tumor-derived TGF- $\beta$ on the balance of tumor growth and immunity. Immunol Lett 2017;191:47-54.

11. Ng C, Li H, Wu WKK, Wong SH, et al. Genomics and metagenomics of colorectal cancer. J Gastrointest Oncol 2019;10:1164-70.

12. Qi H, Liu Y, Qi X, et al. Dietary Recombinant Phycoerythrin Modulates the Gut Microbiota of H22 Tumor-Bearing Mice. Mar Drugs 2019;17:665.

13. Zhao Y, Jiang Q. Roles of the Polyphenol-Gut Microbiota Interaction in Alleviating Colitis and Preventing ColitisAssociated Colorectal Cancer. Adv Nutr 2020. [Epub ahead of print]. doi: 10.1093/advances/nmaa104.

14. Oh NS, Lee JY, Kim YT, et al. Cancer-protective effect of a synbiotic combination between Lactobacillus gasseri 505 and a Cudrania tricuspidata leaf extract on colitis-associated colorectal cancer. Gut Microbes 2020;12:1785803.

15. Vivarelli S, Salemi R, Candido S, et al. Gut Microbiota and Cancer: From Pathogenesis to Therapy. Cancers (Basel) 2019;11:38.

16. Fahmy CA, Gamal-Eldeen AM, El-Hussieny EA, et al.

Cite this article as: Shang F, Jiang X, Wang H, Chen S, Wang X, Liu Y, Guo S, Li D, Yu W, Zhao Z, Wang G. The inhibitory effects of probiotics on colon cancer cells: in vitro and in vivo studies. J Gastrointest Oncol 2020;11(6):1224-1232. doi: 10.21037/jgo-20-573
Bifidobacterium longum Suppresses Murine Colorectal Cancer through the Modulation of oncomiRs and Tumor Suppressor miRNAs. Nutr Cancer 2019;71:688-700.

17. An BC, Hong S, Park HJ, et al. Anti-Colorectal Cancer Effects of Probiotic-Derived p8 Protein. Genes (Basel) 2019;10:624.

18. Escamilla J, Lane MA, Maitin V. Cell-free supernatants from probiotic Lactobacillus casei and Lactobacillus rhamnosus GG decrease colon cancer cell invasion in vitro. Nutr Cancer 2012;64:871-8.

19. Costanzo M, Cesi V, Palone F, et al. Krill oil, vitamin $\mathrm{D}$ and Lactobacillus reuteri cooperate to reduce gut inflammation. Benef Microbes 2018;9:389-99.

20. Zitvogel L, Daillère R, Roberti MP, et al. Anticancer effects of the microbiome and its products. Nat Rev Microbiol 2017;15:465-78.

21. Shuwen H, Xi Y, Quan Q, et al. Relationship between intestinal microorganisms and T lymphocytes in colorectal cancer. Future Oncol 2019;15:1655-66.

(English Language Editor: J. Teoh) 